\title{
Atividade antimicrobiana dos extratos metanólicos da raiz, caule e folhas de Croton pulegioides Baill. (Zabelê)
}

\begin{abstract}
ARRAIS, L.G. ${ }^{1}$; LYRA, H.F.S.'; BATISTA, D.C.A. ${ }^{3}$; COUTINHO, F.N. ${ }^{3}$; SARAIVA, A.M. ${ }^{3}$; PEREIRA, R.C.A.4; PISCIOTTANO, M.N.C. ${ }^{3}$; XAVIER, H.S. ${ }^{2}$; MELO, S.J. ${ }^{1 *}$

'Laboratório de Síntese e Química de Produtos Naturais, Departamento de Antibióticos da Universidade Federal de Pernambuco (UFPE) - Av. Prof. Arthur de Sá, s/n, Cidade Universitária 54740-521 - Recife - PE, Brasil; 'Laboratório de Farmacognosia do Depto. de Ciências Farmacêuticas da Universidade Federal de Pernambuco (UFPE) - Av. Prof. Arthur de Sá, s/n, Cidade Universitária 54740-521 - Recife - PE, Brasil ; '3Laboratório de Análises Microbiológicas do Depto. De Ciências Farmacêuticas da Universidade de Pernambuco (UFPE) - Av. Prof. Arthur de Sá, s/n, Cidade Universitária 54740-521 - Recife - PE, Brasil; " Instituto Agronômico de Pernambuco - Av. Gen. San Martin, 1371 Bongi 50761-000 - Recife - PE - Brasil. *melosebastiao@pq.cnpq.br/maria.nelly@ pq.cnpq.br
\end{abstract}

RESUMO: O uso popular de plantas medicinais continua sendo de grande interesse para a população, principalmente em países em desenvolvimento, nos quais o acesso à medicina alopática ainda é muito restrito. Assim, além de sua importância na medicina tradicional, o conhecimento das plantas pode fornecer para a indústria farmacêutica, direta ou indiretamente, princípios ativos de interesse terapêutico. No presente trabalho foi avaliada a atividade antimicrobiana dos extratos metanólicos secos de Croton pulegioides Baill. obtidos a partir da raiz, do caule e das folhas nas concentrações de 25,50 e $100 \mathrm{mg} / \mathrm{mL}$ utilizando o método de difusão em Agar (técnica de poços) frente a cinco cepas Gram-positivas, três cepas de fungos e três cepas de bactérias Gram-negativas. Na execução do estudo foi utilizado o antibiótico Gentamicina $(100 \mu \mathrm{g} / \mathrm{mL})$ no teste com bactérias, e Cetoconazol $(300 \mu \mathrm{g} / \mathrm{mL})$ para as Candidas verificando-se, ao mesmo tempo, a ausência de atividade para o diluente DMSO $20 \% \mathrm{em}$ água. Na observação dos resultados evidenciaram-se halos de inibição indicando atividade dos extratos metanólicos da raiz, caule e folhas, nas concentrações de 50 e $100 \mathrm{mg} / \mathrm{ml}$, frente Bacillus subtilis AM 04; dos extratos metanólicos da raiz e do caule, nas concentrações de 50 e $100 \mathrm{mg} / \mathrm{ml}$, frente Staphylococcus aureus AM 103 (ATCC 6538); e do extrato metanólico do caule, nas concentrações de 50 e $100 \mathrm{mg} / \mathrm{ml}$, frente S. epidermidis AM 235. Por outro lado, todos os extratos foram inativos nas concentrações avaliadas frente às bactérias Gram-negativas utilizadas no estudo (Pseudomonas aeruginosa AM 206, Escherichia coli AM 1050 e Klebsiella pneumoniae AM 410). Referente à atividade sobre fungos, os extratos metanólicos da raiz e do caule inibiram, nas três concentrações avaliadas, o crescimento de Candida tropicalis AM 1181 e C. albicans AM 1140, enquanto o extrato das folhas, inibiu o crescimento de $C$. tropicalis AM 1181 nas concentrações de 50 e 100mg/ml e de C. albicans AM 1140 apenas na concentração de $100 \mathrm{mg} / \mathrm{mL}$. Finalmente o extrato metanólico das folhas, na concentração de $100 \mathrm{mg} / \mathrm{mL}$, foi o único a inibir o crescimento de Candida krusei. Os resultados preliminares apresentados pelo presente trabalho sugeriram que a espécie Croton pulegioides Baill. apresentou resultados promissores quanto ao potencial antimicrobiano.

Palavras-chave: Plantas medicinais, Croton pulegioides Baill., Atividade antimicrobiana.

ABSTRACT: Antimicrobial activity of methanol extracts of root, stem and leaves of Croton pulegioides Baill. The employment of medicinal plants as a folk custom remains important to the present time, mainly in developing countries, where access to allopathic medicine is restricted. Besides their importance in traditional medicine, the knowledge on these plants can, directly or indirectly, provide information about the active ingredients involved, which may be of interest to the pharmaceutical industry. The present study evaluated the antimicrobial activity of dried methanolic extracts of Croton pulegioides Baill, extracted from its roots, stalk, and leaves, at the concentrations of 25,50 and $100 \mathrm{mg} / \mathrm{ml}$. These were diffused in wells with an Agar medium, to which five strains of Gram-positive, three strains of fungi and three strains of 
Gram-negative bacteria had been added. For this study, the antibiotic Gentamicin $(10 \mu \mathrm{g} / 100 \mu \mathrm{L})$ was used in tests with bacteria, and Ketoconazole $(30 \mu \mathrm{g} / 100 \mu \mathrm{L})$, with Candidas. When DMSO diluted in $20 \%$ water was used, no activity was observed. The results produced evidence of inhibition halos, which indicates the activity of the methanolic extracts from the roots, stalk and leaves at the concentrations of 50 and $100 \mathrm{mg} / \mathrm{ml}$ for Bacillus subtilis AM 04; activity of the methanolic extracts from the roots and stalk at the concentrations of 50 and $100 \mathrm{mg} / \mathrm{ml}$ for Staphylococcus aureus AM 103 (ATCC 6538); and activity of the methanolic extract from the stalk at the concentrations of 50 and $100 \mathrm{mg} / \mathrm{ml}$ for Staphylococcus epidermidis AM 235. There was no activity in the concentrations studied for the Gram-negative bacteria (Pseudomonas aeruginosa AM 206, Escherichia coli AM 1050 and Klebsiella pneumoniae AM 410). With respect to fungal activity, the methanolic extracts from the roots and stalk inhibited Candida tropicalis AM 1181 and C. albicans AM 1140 at the three concentrations studied. The extract from the leaves inhibited the growth of $C$. tropicalis AM 1181 at the concentrations of 50 e $100 \mathrm{mg} / \mathrm{ml}$ and C. albicans AM 1140 only at the concentration of $100 \mathrm{mg} / \mathrm{ml}$. Finally, the methanolic extract from the leaves, at the concentration of $100 \mathrm{mg} / \mathrm{ml}$, was the only one to inhibit the growth of Candida krusei. Preliminary results from this study suggest that the Croton pulegioides Baill showed promising results as a potential antimicrobial agent.

Keywords: Medicinal plants; Croton pulegioides Baill. ; Microbial Activity.

\section{INTRODUÇÃO}

O uso de plantas medicinais é feito desde a antiguidade e o conhecimento sobre as suas propriedades representa, muitas vezes, o único recurso terapêutico de várias comunidades e grupos étnicos. As observações populares contribuem para a divulgação destes vegetais pelos efeitos medicinais que produzem, apesar de que, geralmente, não se conheça seus constituintes químicos e se torne válidas as informações acumuladas ao longo dos anos (Maciel et al., 2002).

Newman \& Cragg (2012) realizaram uma ampla revisão referente à origem de novas drogas aprovadas para uso humano em diversas patologias nos últimos 30 anos, destacando a importância de produtos naturais e suas estruturas químicas.

Assim, tendo em vista o potencial terapêutico de plantas medicinais, a ampliação da produção científica nesta área é de grande importância para aprofundar o conhecimento de suas atividades farmacológicas e de suas propriedades toxicológicas (Calixto, 2000; Barros, 2008).

O Brasil é detentor da maior biodiversidade do planeta - conta com cerca de 55.000 das 400.000 espécies de plantas existentes, e possui numerosas espécies produtoras de substâncias bioativas (Albuquerque et al., 2007). Com uma área territorial de 8,5 milhões de quilômetros quadrados e vários biomas (Mata Atlântica, Cerrado, Pantanal, Amazônia e Caatinga), apresenta grande diversidade de solos e climas que favorece a riqueza e variedade de tipos de vegetação e espécies de flora distribuída nos diversos ecossistemas brasileiros (Portal Brasil, 2010).

No Semi-Árido brasileiro, com extensão de 868 mil quilômetros quadrados, observa-se crescente interesse pelo estudo das plantas medicinais da região (Albuquerque et al., 2007), uma vez que são potencialmente ricas em propriedades curativas, sendo ainda pouco estudadas do ponto de vista taxonômico, químico, além do poder bioativo de suas moléculas (Cunha et al., 2003; David et al., 2006).

Neste contexto, a mata Atlântica no Estado de Pernambuco apresenta diferentes grupos vegetais ainda pouco estudados, dentre os quais figura o gênero Croton, que pertence à família Euphorbiaceae, a qual compreende cerca de 320 gêneros e 8700 espécies (Steinmann, 2002).

O gênero Croton é representado por árvores, arbustos e ervas, difundidos em zonas tropicais e subtropicais das Américas, África e Ásia (Govaerts, 2000).

A química do gênero Croton é diversa, fato que qualifica o grupo como altamente promissor para estudos de prospecção de substâncias naturais farmacologicamente ativas, sendo, por este motivo, alvo de pesquisas tanto em estudos fitoquímicos, como por suas atividades biológicas (Randau et al., 2004).

A composição química das espécies do gênero Croton é rica em metabólitos secundários, tais como, alcaloides, flavonoides e terpenoides, (Randau et al., 2004; Payo, et al., 2001). Além destes metabólitos, muitas das espécies de Croton produzem óleos essenciais ricos em mono e sesquiterpenoides, como também fenilpropanoides (Palmeira et al. 2006).

Pessoa, et al. (2012), também relataram a presença de metabólitos secundários de natureza terpênica e fenólica na espécie Croton

Rev. Bras. PI. Med., Campinas, v.16, n.2, supl. I, p.316-322, 2014. 
pedicellatus Kunth, a qual pode ser confirmada pelo isolamento de dois análogos do tilirosídeo, 6 "-O-p-cumaroil- $\beta$-galactopiranosilcanferol e 6"-O- $p$ cumaroil- $\beta$-glicopiranosil-3'-metoxicanferol, além da alpinumisoflavona, 3-glicopiranosilquercetina e do ácido 4-hidroxi-3,5-dimetoxibenzoico.

Um estudo fitoquímico realizado a partir das cascas da espécie Croton celtidifolius Baill. , descreveu a presença de elevado teor de compostos fenólicos totais, proantocianidinas totais e baixo teor de flavonoides (Horst, 2008).

No uso popular, as espécies do Croton são utilizadas sob a forma de chás e infusões para o alívio de dores (Abreu, 2001), tratamento de distúrbios digestivos tais como: constipações e diarreias, e também como antiinflamatórios e antiulcerogênicos, analgésicos, antidiabéticos e antimaláricos. Além disso, segundo Morais, 2006, três espécies originárias do Ceará (C. zenhtneri, C. nepetaefolius e C.argyrophylloides) foram recentemente confirmadas como antioxidantes devido à ação de sete diferentes constituintes de seus óleos essenciais.

Outras espécies de Croton, também já tiveram suas atividades avaliadas e confirmadas, como o caso do Croton lechleri, para o qual foram descobertas atividades antioxidante, antimicrobiana e antiviral (Gupta et. al., 2008), as atividades anti-helmíntica e efeito cardiovascular do Croton zehntneri (Camurça-Vasconcelos et al., 2007, De Siqueira et al., 2006), antinociceptiva do Croton urucurana (Rao et al., 2007), moluscicida do Croton campestris (Babili et al., 2006), anti-inflamatória do Croton cuneatus (Suárez et al., 2006), e mutagênica e antioxidante do Croton lechleri (Lopes e Lopes et al., 2004).

O gênero Croton possui algumas espécies com atividade antimicrobiana, podendo-se citar uma substância isolada do caule de Croton jacobinensis Baill. , com ação sobre cepas de Neisseria, de Nocardia, e de Brucella (Lima et. al., 2001); e a ação dos diterpenos isolados da espécie Croton nepetaefolius, sobre cepas de Streptococcus sp. oriundos da mucosa oral e produtores de biofilme (Teixeira et al., 2011).

A espécie Croton pulegioides Baill. , conhecida popularmente como "Zabelê", foi objeto de poucos estudos fitoquímicos, havendo dentre eles, uma pesquisa em que se relata a presença de constituintes voláteis (Da Camara \& Neves, 2012).

Tendo em vista o potencial terapêutico e econômico do gênero, o presente trabalho buscou a ampliação do conhecimento sobre a espécie Croton pulegioides Baill, espécie ainda não estudada quanto à sua atividade antimicrobiana.

\section{MATERIAL E MÉTODOS}

\section{Coleta do material}

A planta fresca foi coletada no município de Gravatá - PE - Brasil, no período compreendido entre novembro de 2010 e setembro de 2011. O material botânico foi identificado pela $\operatorname{Prof}^{\mathrm{a}} \mathrm{Dr}^{\mathrm{a}}$. Rita de Cássia Araújo Pereira (curadora do Herbário IPA) e exsicata depositada no Herbário Dárdano de Andrade Lima, Instituto Agronômico de Pernambuco, sob o número 80985.

\section{Obtenção dos extratos}

Os extratos foram preparados a partir da raiz, caule e folhas do Croton pulegioides Baill, usando respectivamente $20 \mathrm{~g}, 70 \mathrm{~g}$ e $50 \mathrm{~g}$ do vegetal seco. O material vegetal seco e moído foi submetido a uma extração por maceração usando metanol como solvente. Seguidamente, o solvente foi filtrado e o procedimento de extração foi repetido por mais duas vezes. A solução foi destilada à pressão reduzida em evaporador rotativo obtendo-se os rendimentos de $16 \%$ para o extrato bruto seco da raiz, $9 \%$ para o extrato bruto seco das folhas, e 10\% para o extrato bruto seco do caule.

\section{Microorganismos}

$\mathrm{Na}$ determinação da atividade antimicrobiana foram utilizadas 11 (onze) cepas pertencentes à "Coleção de Culturas do Laboratório de Análises Microbiológicas (AM)" do Departamento de Ciências Farmacêuticas da Universidade Federal de Pernambuco (UFPE), das quais, 8 (oito) bactérias: Staphylococcus aureus AM 103 (ATCC 6538), Staphylococcus epidermidis AM 235, Staphylococcus saprophyticus AM 245, Enterococcus faecalis AM 1056, Pseudomonas aeruginosa AM 206, Escherichia coli AM 1050, Klebsiella pneumoniae AM 410, Bacillus subtilis AM 04, e três cepas de fungos: Candida krusei AM 1168, Candida tropicalis AM 1181, Candida albicans AM 1140.

\section{Avaliação da atividade antimicrobiana}

A atividade antimicrobiana do extrato seco metanólico de Croton pulegioides foi determinada pela metodologia de Técnica de Poços - Difusão em Agar. No teste, as culturas microbianas foram preparadas a partir de colônias isoladas das cepas reativadas e suspendidas em $5 \mathrm{~mL}$ de solução salina estéril. A turvação da suspensão microbiana foi padronizada comparando-se com a escala 0,5 de MacFarland, a qual corresponde à concentração de

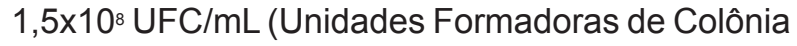
por mililitro), semeando cada suspensão microbiana com o auxílio de um swab estéril sobre a superfície da placa contendo $20 \mathrm{~mL}$ de Agar Mueller Hinton (bactérias) ou Agar Sabouraud (fungos) para obter

Rev. Bras. PI. Med., Campinas, v.16, n.2, supl. I, p.316-322, 2014. 
um tapete homogêneo, e furando seguidamente os meios até o fundo da placa com um perfurador estéril para obter os poços de $6 \mathrm{~mm}$ de diâmetro (5 poços por placa) que vão receber cada um 100 microlitros das respectivas soluções:os extratos em três diferentes concentrações; a solução do antibiótico padrão, gentamicina ou cetoconazol, e o diluente utilizadoDMSO/ $\mathrm{H}_{2} \mathrm{O}$. Assim, as concentrações dos extratos em teste foram $100 \mathrm{mg} /$ $\mathrm{mL}$ (ou $10 \mathrm{mg} / 100 \mu \mathrm{L}$ ), $50 \mathrm{mg} / \mathrm{mL}$ (ou $5 \mathrm{mg} / 100 \mu \mathrm{L}$ ) e $25 \mathrm{mg} / \mathrm{mL}$ (ou 2,5 mg/100 $\mu \mathrm{L}$ ), respectivamente; no quarto poço foi introduzida a concentração do antibiótico Gentamicina 0,1 mg/mL (ou $10 \mu \mathrm{g} / 100$ $\mu \mathrm{L}$ ), ou Cetoconazol 0,3 mg/mL (ou $30 \mu \mathrm{g} / 100 \mu \mathrm{L}$ ), deixando o quinto poço para o controle do diluente (DMSO/ $\mathrm{H}_{2} \mathrm{O}$ à $20 \%$ ). As placas foram incubadas por 18 horas à $37^{\circ} \mathrm{C} \pm 1^{\circ}$, medindo-se os halos de inibição produzidos, sendo realizado todos os ensaios em triplicata.

\section{RESULTADOS E DISCUSSÃO}

Tendo em vista o problema da resistência das bactérias a múltiplos antimicrobianos, revelouse a importância do conhecimento de espécies vegetais com propriedades antimicrobianas e, consequentemente, a busca por substâncias derivadas de plantas teve grande impulso nos últimos anos (Coelho et. al., 2004). O interesse pelas plantas é devido em parte, à grande variedade de substâncias químicas pertencentes a diferentes classes de metabolitos secundários, tais como alcalóides, flavonóides, terpenóides e compostos fenólicos, dos quais, muitos deles, possuem potente atividade antimicrobiana já demonstrada por pesquisas (Nascimento, 2000).

Os resultados da atividade antimicrobiana dos extratos metanólicos de Croton pulegioides Baill são apresentados nas Tabelas 1 e 2.

TABELA 1. Atividade antimicrobiana do extrato metanólico seco da Raiz, do Caule e das Folhas de Croton pulegioides Baill. .

\begin{tabular}{|c|c|c|c|c|c|}
\hline \multirow[b]{2}{*}{ Microorganismo } & \multirow{2}{*}{$\begin{array}{c}\text { Concentração } \\
\text { de extrato }{ }^{1}\end{array}$} & \multicolumn{3}{|c|}{ Halos de inibição (mm) } & \multirow{2}{*}{$\begin{array}{c}\text { GENTAMICINA }^{5} \\
\text { Halos de inibição }(\mathrm{mm})\end{array}$} \\
\hline & & ExMR $^{2}$ & ExMC $^{3}$ & ExMF $^{4}$ & \\
\hline \multirow[t]{3}{*}{ Bacillus subtilis $\mathrm{AM}^{6} 04$} & 10 & 11 & 13 & 13 & (25), (31), (32) \\
\hline & 5 & 10 & 12 & 10 & \\
\hline & 2,5 & $(-)$ & $(-)$ & $(-)$ & \\
\hline \multirow[t]{3}{*}{ Staphylococcus aureus AM 103} & 10 & 14 & 16 & $(-)$ & (24), (23), (27) \\
\hline & 5 & 11 & 12 & $(-)$ & \\
\hline & 2,5 & $(-)$ & $(-)$ & $(-)$ & \\
\hline Staphylococcus epidermidis & 10 & $(-)$ & 15 & $(-)$ & (22), (25), (27) \\
\hline \multirow[t]{2}{*}{ AM 235} & 5 & $(-)$ & 11 & $(-)$ & \\
\hline & 2,5 & $(-)$ & $(-)$ & $(-)$ & \\
\hline Staphylococcus saprophyticus & 10 & $(-)$ & $(-)$ & $(-)$ & $(22),(25),(26)$ \\
\hline \multirow[t]{2}{*}{ AM 245} & 5 & $(-)$ & $(-)$ & $(-)$ & \\
\hline & 2,5 & $(-)$ & $(-)$ & $(-)$ & \\
\hline \multirow[t]{3}{*}{ Enterococcus faecalis AM 1056} & 10 & $(-)$ & $(-)$ & $(-)$ & (22), (13), (17) \\
\hline & 5 & $(-)$ & $(-)$ & $(-)$ & \\
\hline & 2,5 & $(-)$ & $(-)$ & $(-)$ & \\
\hline Pseudomonas aeruginosa & 10 & $(-)$ & $(-)$ & $(-)$ & $(22),(23),(23)$ \\
\hline \multirow[t]{2}{*}{ AM 206} & 5 & $(-)$ & $(-)$ & $(-)$ & \\
\hline & 2,5 & $(-)$ & $(-)$ & $(-)$ & \\
\hline \multirow[t]{3}{*}{ Escherichia coli AM 1050} & 10 & $(-)$ & $(-)$ & $(-)$ & (19), (18), (27) \\
\hline & 5 & $(-)$ & $(-)$ & $(-)$ & \\
\hline & 2,5 & $(-)$ & $(-)$ & $(-)$ & \\
\hline \multirow[t]{3}{*}{ Klebsiella pneumonia AM 410} & 10 & $(-)$ & $(-)$ & $(-)$ & $(17),(22),(26)$ \\
\hline & 5 & $(-)$ & $(-)$ & $(-)$ & \\
\hline & 2,5 & $(-)$ & $(-)$ & $(-)$ & \\
\hline
\end{tabular}

Legenda: (-) = inativo; 1 = mg/100 $\mu \mathrm{L}$ (a solução diluente empregada - DMSO 20\% - não interferiu no crescimento bacteriano); 2 = ExMR (Extrato seco Metanólico da Raiz); 3 = ExMC (Extrato seco Metanólico do Caule); 4 = ExMF (Extrato seco Metanólico das Folhas); 5 = antibiótico padrão (As três leituras para cada cepa correspondem aos valores obtidos nos ensaios feitos com cada um dos três extratos); 6 = AM: Coleção de cepas do Laboratório de Análises Microbiológicas, Departamento de Ciências Farmacêuticas, UFPE. 
TABELA 2. Atividade antifúngica do extrato metanólico seco da raiz de C. pulegioides Baill. .

\begin{tabular}{|c|c|c|c|c|c|}
\hline \multirow[t]{2}{*}{ Microorganismo } & \multirow{2}{*}{$\begin{array}{c}\text { Concentração } \\
\text { de extrato }{ }^{1}\end{array}$} & \multicolumn{3}{|c|}{ Halos de inibição $(\mathrm{mm})$} & \multirow{2}{*}{$\begin{array}{c}\text { CETOCONAZOL }{ }^{5} \\
\text { Halos de inibição }(\mathrm{mm})\end{array}$} \\
\hline & & ExMR $^{2}$ & ExMC $^{3}$ & ExMF ${ }^{4}$ & \\
\hline \multirow[t]{3}{*}{ Candida albicans AM 1140} & 10 & 23 & 13 & 13 & \multirow[t]{3}{*}{ (15), (15), (15) } \\
\hline & 5 & 17 & 12 & 10 & \\
\hline & 2,5 & 14 & $(-)$ & $(-)$ & \\
\hline \multirow[t]{3}{*}{ Candida krusei AM 1168} & 10 & $(-)$ & $(-)$ & 12 & \multirow[t]{3}{*}{ (22), (22), (22) } \\
\hline & 5 & $(-)$ & $(-)$ & $(-)$ & \\
\hline & 2,5 & $(-)$ & $(-)$ & $(-)$ & \\
\hline \multirow[t]{3}{*}{ Candida tropicalis AM 1181} & 10 & 23 & 22 & 14 & \multirow[t]{3}{*}{ (15), (15), (15) } \\
\hline & 5 & 19 & 17 & 12 & \\
\hline & 2,5 & 16 & 13 & $(-)$ & \\
\hline
\end{tabular}

Legenda: $(-)$ = inativo; 1 = mg/100 $\mu \mathrm{L}$ (a solução diluente empregada - DMSO 20\% - não interferiu no crescimento bacteriano); 2 = ExMR (Extrato seco Metanólico da Raiz); 3 = ExMC (Extrato seco Metanólico do Caule); 4 = ExMF (Extrato seco Metanólico das Folhas); 5 = antifúngico padrão (As três leituras para cada cepa correspondem aos valores obtidos nos ensaios feitos com cada um dos três extratos); 6 = AM: Coleção de cepas do Laboratório de Análises Microbiológicas, Departamento de Ciências Farmacêuticas, UFPE.

A atividade antibacteriana apresentada na Tabela 1, destaca-se os halos obtidos com o extrato seco metanólico da raiz frente à cepa de Staphylococcus aureus AM 103 (ATCC 6538) com halo de $14 \mathrm{~mm}$ para a maior concentração testada $(10 \mathrm{mg} / 100 \mu \mathrm{L}$ ou $100 \mathrm{mg} / \mathrm{mL}$ ). No caso do extrato seco metanólico do caule, houve atividade para as cepas de Staphylococcus aureus AM 103 e Staphylococcus epidermidis AM 235 (halos da ordem de $15 \mathrm{~mm}$ para a concentração de $100 \mathrm{mg} /$ $\mathrm{mL}$ ), enquanto não se observou atividade do extrato seco metanólico das folhas.

Referente ao tamanho dos halos, Alves (2000) propôs uma classificação da ação para os extratos, na qual se considera como inativo aquele produz um halo menor que $9 \mathrm{~mm}$; enquanto que resultados de 9 a $12 \mathrm{~mm}$ indicam extratos ativos, e finalmente halos de 13 a $18 \mathrm{~mm}$, ou maiores, correspondem a extratos muito ativos.

Do que antecede, podemos considerar promissores estes primeiros resultados com extratos metanólicos do Croton pulegioides Baill. , destacando ainda a importância de sua atividade sobre microrganismos Gram-positivos, especialmente Staphylococcus aureus.

A prospecção fitoquímica indica que o gênero Croton apresenta em sua composição, alcaloides, flavonoides, triterpenoides e uma grande variedade estrutural de diterpenoides, os quais podem estar relacionados com essa atividade antimicrobiana contra diversos microorganismos (Urzúa, 2008).

Estudos anteriores assinalaram haver, para o óleo essencial das folhas de Croton zehntneri, uma atividade antimicrobiana importante contra Staphylococcus aureus, entre outros microrganismos (Costa et. al. 2008). Enquanto que para Croton urucurana, a atividade antimicrobiana foi relatada para diversas cepas bacterianas (Oliveira et. al., 2008).

Desta forma, a atividade antimicrobiana observada para os extratos do C. pulegioides Baill. pode ser devida à presença de compostos como flavonoides, terpenos ou taninos reconhecidos com atividade para uma ampla variedade de microrganismos (Ho et al., 2001; Rajan et al., 2011).

No que concerne aos resultados com os isolados de Candida sp. testados (Candida albicans AM 1140 - origem: urina; Candida tropicalis AM 1181 - origem: swab retal; e Candida krusei AM 1168 - origem: sangue), conforme a "Guideline method for Antifungal Disk Diffusion Susceptibility Testing of Yeasts" (NCCLS, 2004), os halos obtidos de $15 \mathrm{~mm}$ correspondem a um caráter sensível de dose-dependente.

Com relação à cepa de Candida krusei, a qual apresentou um halo maior frente ao antifúngico padrão utilizado nos ensaios, é considerado um patógeno oportunista (Pfaller et al., 2010), com características de sensibilidade variáveis com a origem.

\section{CONCLUSÃO}

A determinação da atividade antimicrobiana da espécie C. pulegioides Baill. indicou que os maiores halos de inibição foram frente aos fungos e às bactérias Gram-positivas. Esta diferença de ação pode estar relacionada não só aos próprios produtos testados, como também devido à estrutura mais complexa da membrana externa das bactérias Gram-negativas, que pode impedir a passagem de moléculas (França et al., 2009), além de particularidades relacionadas aos diferentes

Rev. Bras. PI. Med., Campinas, v.16, n.2, supl. I, p.316-322, 2014. 
mecanismos de resistência das linhagens em estudo.

Os resultados preliminares obtidos pelo presente trabalho, conforme a classificação proposta por Alves (2000), sugerem que a espécie $C$. pulegioides Baill. , apresenta resultados promissores quanto ao potencial antimicrobiano.

\section{REFERÊNCIA}

Abreu, A.S. et al. Constituintes químicos do caule e das cascas do caule de Croton pullei var Glabrior (Euphorbiaceae). Revista Virtual de Iniciação Científica UFPA, v.1, n.2, p. 1-9, 2001.

Albuquerque, U.P. et al. Medicinal plants of the caatinga (semi-arid) vegetation of NE Brazil: a quantitative approach. Journal of Ethnopharmacology, v.14, p.325-54, 2007.

Alves, T.M.A. et al. Biological screening of Brazilian Medicinal Plants, Memórias do Instituto Oswaldo Cruz, v.95, p.367-73, 2000.

Babili, F. E. L. et al. Molluscicidal activity against Bulinus truncatus of Croton campestris. Fitoterapia, v.77, p.384-387, 2006.

Barros, F. M. C. Variabilidade sazonal, atividade antimicrobiana, fracionamento bio-guiado, isolamento e elucidação estrutural dos principais constituintes do óleo essencial de Lippia Alba (MILL.) N. E. Brown. 2008. 162p. Dissertação (Mestrado - Área de concentração em Ciências Farmacêuticas) - Universidade Federal de Santa Maria, Rio Grande do Sul.

Calixto, J.B. Eficacy, safety, quality control, marketing and regulatory guidelines for herbal medicines (phytotherapeutic agents). Brazilian Journal and Biological Research, v.33, p.179-89, 2000.

Camurça-Vasconcelos, et al. Anthelmintic activity of Croton zehntneri and Lippia sidoides essential oils. Veterinary Parasitology, v.148, n. 3-4, p.288-294, 2007.

Coelho, G.S. et al. Etnopharmacological studies of antimicrobial remedies in the South of Brazil. Journal of Ethnopharmacology,v.90, p.135-43, 2004.

Cunha, A.P.; Silva, A.P.; Roque, O.R. Plantas e produtos vegetais em fitoterapia. Lisboa: Calouste Gulbenkian, p. 21-61, 2003.

Costa, J.G.M. et al. Composição química e avaliação da atividade antibacteriana e toxicidade do óleo essencial de Croton zehntneri (variedade estragol). Revista Brasileira de Farmacognosia, v.18, p.583-86, 2008.

Da Camara, C. A. G.; Neves, I. A. . Volatile Constituents of Two Croton Species from Caatinga Biome of Pernambuco - Brasil. Rec. Nat. Prod. 6:2, 161-165, 2012

David, J.P.L. et al. Introdução. In Lucchese AM (ed.). Instituto do Milênio do Semi-árido. Plantas da Caatinga: perfil botânico, fitoquímica e atividade biológica. Associação de Plantas do Nordeste, v.4, p.15-7, 2006.

De Siqueira, R. J. B. et al. Cardiovascular effects of the essential oil of Croton zehntneri leaves and its main constituents, anethole and estragole, in normotensive conscious rats. LifeSciences, v.78, n.20, p.2365-2372, 2006.
França, H.S. et al. Atividade antibacteriana de floroglucinóis e de extrato hexânico de Hypericum brasiliense Choysi. Química Nova, v.32, p.1103-6, 2009.

Govaerts, R.; Frodin, D.G.; Radcliffe-smith, A. Croton. In: World Checklist and bibliography of Euphorbiaceae (and Pandaceae.) Royal Botanic Gradens Kew, Lodon. v.2, p.417-536, 2000.

Gupta, D.; Blekley, B.; Gupta, R.K. Dragons blood: botany, chemistry and therapeutic uses. Journal of ethnopharmacology, v.115, p. 361-80, 2008.

Ho, K.Y. et al. Antimicrobial activity of tannin components from Vaccinium vitisidaea L. Journal of Pharmacy and Pharmacology, v.53, p.187-1, 2001.

Horst, H. ANÁLISE QUÍMICA E BIOLÓGICA DOS CONSTITUINTES FENÓLICOS DE Croton celtidifolius Baill. Dissertação (Mestrado - Programa de PósGraduação em Química - Universidade Federal de Santa Catarina), 2008.

Lima, E. O. Plantas e suas propriedades antimicrobianas: uma breve analise histórica. In: YUNES, R. A.; CALIXTO, J. B. (Orgs.). Plantas medicinais: sob a óptica da química medicinal moderna. Chapecó: ARGOS, p. 483-501, 2001.

LOPES E LOPES, M. I. et al. Mutagenic and antioxidant activities of Croton lechleri sap in biological systems. Journal of Ethnopharmacology, v.95, n.2-3, p.437445, 2004.

Maciel, M.A.M. et al. Plantas medicinais: a necessidade de estudos multidisciplinares. Química Nova, v.25, p.429-38, 2002.

Morais, S.M. et al. Atividade antioxidante de óleos essências de espécies de Croton do nordeste do Brasil. Quimica Nova, v.29, p.907-10, 2006.

Nascimento, G.G.F. et al. Antibacterial activity of extracts and phytochemicals antibiotic-resistant bacteria. Brazilian Journal of Microbiology, v.31, p.247-56, 2000.

NCCLS. Method for Antifungal Disk Diffusion Susceptibility Testing of Yeasts; Approved Guideline. NCCLS document M44-A, 2004.

Newman, D.J. and Cragg G.M. Natural Products As Sources of New Drugs over the 30 Years from 1981 to 2010. Journal of Natural Products, v. 75, p. 311-35, 2012.

Oliveira, I.S. et al. Triagem da atividade antibacteriana in vitro do látex e extratos de Croton urucurana Baillon. Revista Brasileira de Farmacognosia, v.18, p. 58793, 2008.

Palmeira, Jr.S.F. et al. Constituintes químicos das folhas de Croton sellowii (Euphorbiaceae). Revista Brasileira de Farmacognosia, v.16, n.3, p.397-402, 2006.

Pessoa, O. D. L. et al. . Flavonoides e sesquiterpenos de Croton pedicellatus Kunth. . Química Nova, Vol. 35, No. 11, 2169-2172, 2012.

Payo, H.A. et al. Tamizaje fitoquímico preliminar de espécies del género Croton L. Revista Cubana de Farmácia, v.35, p.203-06, 2001.

Pfaller, M.A. et al.. Results from the ARTEMIS DISK Global Antifungal Surveillance Study, 1997 to 2007: a 10.5Year Analysis of Susceptibilities of Candida Species to Fluconazole and Voriconazole as Determined by CLSI Standardized Disk Diffusion, J Clin Microbiol. April; 48(4): 1366-1377, 2010.

Portal Brasil. Por dentro do Brasil - Meio Ambiente.

Rev. Bras. PI. Med., Campinas, v.16, n.2, supl. I, p.316-322, 2014. 
34p. Disponível em: <http://www.brasil.gov.br/cop/ materiais-para-download/por-dentro-do-brasil-2013meio-ambiente> Acesso em: 28 mar. 2013.

Rajan, S.; Thirunalasundari, T.; Jeeva, S. Anti-enteric bacterial activity and phytochemical analysis of the seed kernel extract of Mangifera indica Linnaeus against Shigella dysenteriae (Shiga, corrig.) Castellani and Chalmers. Asian Pacific Journal of Tropical Medicine, p.294-300, 2011.

Randau, K.P., et al. Estudo farmacognóstico de Croton rhamnifolius H.B.K. e Croton rhamnifolioides Pax \& Hoffm. (Euphorbiaceae). Revista Brasileira de Farmacognosia, v.4, p.89-96, 2004.

RAO, V. S., et al. Dragon's blood from Croton urucurana
(Baill.) attenuates visceral nociception in mice. Journal of Ethnopharmacology, v.113, n.2, p.357-360, 2007.

Steinmann, V.W. Diversidad y endemismo de la familia Euphorbiaceae em Mexico. Ata Botanica Mexicana Pátzcuaro, v.61, p.61-93, 2002.

Suárez, A. I., et al. Anti-inflammatory activity of Croton cuneatus aqueous extract. Journal of Ethnopharmacology, v.105, n.1-2 2006, p.99-101.

Teixeira E. H., et al. Antimicrobial and antibiofilm action of Casbane Diterpene from Croton nepetaefolius against oral bacteria. Archives of oral biology, v. 57, 550-555, 2012.

Urzúa, A. et al. A Structure-Activity Study of Antibacterial Diterpenoids. Molecules, v.13, 882-91, 2008. 